\title{
Dynamic Joint Resource Optimization for LTE-Advanced Relay Networks
}

\author{
Honghao Ju, Ben Liang, Senior Member, IEEE, Jiandong Li, Senior Member, IEEE, and Xiaoniu Yang
}

\begin{abstract}
A dynamic optimization algorithm is proposed for the joint allocation of subframes, resource blocks, and power in the Type 1 inband relaying scheme mandatory in the LTEAdvanced standard. Following the general framework of Lyapunov optimization, we decompose the original problem into three sub-problems in the forms of convex programming, linear programming, and mixed-integer programming. We solve the last sub-problem in the Lagrange dual domain, showing that it has zero duality gap, and that a primal optimum can be obtained with probability one. The proposed algorithm dynamically adapts to traffic and channel fluctuations, it accommodates both instantaneous and average power constraints, and it obtains arbitrarily near-optimal sum utility of each user's average throughput. Simulation results demonstrate that the joint optimum can significantly outperform suboptimal alternatives.
\end{abstract}

Index Terms-LTE-Advanced networks, relay, power, subframe, $R B$, dynamic optimization.

\section{INTRODUCTION}

The LTE-Advanced standard has specified the usage of relay nodes (RNs) as a cost efficient means to extend the service coverage area of a base station (termed eNB, for evolved NodeB) [1]. Each RN accesses the eNB through a wireless backhaul link (BL). It forwards data to and from some user equipment (UE) through a wireless access link (AL). The basic resource granularity for transmission is a resource block (RB), each consisting of twelve $15 \mathrm{kHz}$ subcarriers and six to seven OFDM symbols. The temporal duration of two RBs is represented by one subframe [2].

To accommodate network operators with only one carrier frequency, support for Type 1 relays is mandatory in the LTEAdvanced standard [1] [3]. In the Type 1 relaying scheme, the backhaul link and the access link share the same carrier frequency, with time-division multiplexing to avoid loop interference between the backhaul link receiving antenna and the access link transmitting antenna. More specifically, in each subframe, simultaneous transmissions in the backhaul link and the access link is not allowed. Therefore, an adaptive subframe allocation scheme between the backhaul and access links is of paramount importance.

This work was supported by National Natural Science Foundation of China (61231008), the National Basic Research Program of China (973 Program) (2009CB320404), National Natural Science Foundation of China (61102057), the Program for Changiiang Scholars and Innovative Research Team in University (PCSIRT) (IRT0852), and 111 Project under grant B08038.

Honghao $\mathrm{Ju}$ and Jiandong $\mathrm{Li}$ are with the Information Science Institute, State Key Laboratory of Integrated Service Networks, Xidian University, Xi' an, Shaanxi, China. Ben Liang is with the Department of Electrical and Computer Engineering, University of Toronto, Ontario, Canada. Xiaoniu Yang is with No. 36 Research Institute of China Electronics Technology Group Corporation, Jiaxing, Zhejiang, China. Honghao Ju was a visiting student at the University of Toronto supported by the China Scholarship Council.
The LTE-Advanced standard supports dynamic scheduling, which concerns the assignment of subframes to the backhaul and access links, and the allocation of RBs to the UEs. In addition, the available transmission power at the eNB and RNs is often limited due to legal and hardware constraints, which presents an additional dimension of flexibility and challenges. The decisions in dynamic scheduling and power allocation are highly correlated. Therefore, joint consideration is necessary to provide a judicious solution to fully exploit the channel and user diversity gain in the system.

In this paper, we study the jointly optimal dynamic allocation of subframes, RBs, and power in a Type 1 relay network. Our objective is to maximize the network utility of the average throughput to all UEs. We consider practical impediments, including instantaneous and average power constraints on the eNB and RNs, as well as the special subframe activation limitations on the backhaul and access links imposed by Type 1 relays. Furthermore, by ignoring subframe allocation, the same solution can also be applied to Type 1a outband relaying [1], which deploys the backhaul and access links with different carriers to avoid loop interference.

This three-dimensional joint subframe, RB, and power optimization problem can be expressed as a mixed-integer program, whose solution typically has prohibitive complexity. We explain in Section II the existing methods for resource allocation in OFDMA relay systems. None of them are applicable to our problem, since they either do not accommodate the practical constraints imposed by LTE-Advanced Type 1 relays, or consider only a subset of the three resource allocation problems, leading to suboptimal solutions.

Instead, we propose an asymptotically optimal algorithm for the joint allocation of all three resources. Our main contributions are as follows:

- We adopt a general Lyapunov optimization framework to jointly optimize subframe, RB, and power allocation, which involves the minimization of a Lyapunov driftplus-penalty function [4]. For the specific setting of our problem, we show that this minimization is decomposable into three sub-problems represented by 1) a convex program, 2) a linear program, and 3) a mixed-integer program. The first two sub-problems are solved using standard approaches.

- For the more challenging third sub-problem, we show that optimality can be preserved by continuity relaxation and Lagrange dual decomposition. We further observe that, after optimizing in the dual domain, the special structure of our solution allows recovering the primal optimal solution with probability one. 
- The above solution results in a $1-O\left(\frac{1}{V}\right)$ utility and $O(V)$ delay tradeoff for the overall optimization problem, for any arbitrary positive $V$. The proposed algorithm accounts for both traffic-level variations and channel fading through dynamic adaptation.

- We further show that the proposed algorithm is amenable to practical implementation, and we demonstrate its performance advantage over suboptimal alternatives through simulation.

The organization of the rest of this paper is as follows. In Section II, we discuss the related work. In Section III, we summarize the LTE-Advanced Type 1 inband relay network and present the problem statement. The dynamic joint subframe, $\mathrm{RB}$, and power allocation scheme is presented in Section IV. We give analytical performance bounds and show simulation performance in Section V. Concluding remarks are given in Section VI.

\section{RELATED WORK}

Various solutions to joint resource allocation in OFDMA relay systems have been proposed in the literature. The link scheduling scheme with equal power allocation in [5] requires reusing RBs between the backhaul and access links, which is not supported in the LTE-Advanced standard. A few recent works in OFDMA relaying study the jointly optimal allocation of power and subchannels in [6]-[9], the joint transmission mode, relay node, and subchannel allocation by enabling the physical layer network coding in [10], and the joint relay strategy, relay node, subchannel, and power allocation in [11]. However, the allocation of subchannels is less dynamic than that of subframes and RBs.

Most studies on resource allocation with LTE-Advanced typed relays consider only a partial set among subframes, RBs and power. For example, [12] proposes a low-complexity link scheduling scheme with performance bound by large deviation theory, while [13] studies the effect of multi-cell interference on resource allocation. Neither work considers adaptive subframe activation between the backhaul and access links.

There are relatively few studies on the subframe allocation of inband relaying. Joint subframe and RB allocation for the Type 1 inband relay network has been studied in [14]-[17]. However, none considers power allocation, even though it is supported in the standard [18]. In addition, by reducing the design complexity in the power dimension, they usually face a linear integer optimization problem, which is generally hard to solve. Worse, the additional consideration for joint power allocation changes the problem to non-linear mixed-integer optimization. In particular, the integer variables would exhibit a multiplication form as shown in Section III, which is combined with the non-linear power term leading to drastically increased computational complexity. To the best of our knowledge, no solution exists in the literature for jointly optimal allocation of all three resources.

Related to Lyapunov-typed optimization with data forwarding by relays, the backpressure-typed algorithms have been proposed to dynamically schedule packet transmission over multiple hops [19]-[21]. The admission control component of our algorithm borrows from this general approach. However, these works generally assume a given function that maps channel state to data rate, without considering how to optimize the actual system details, such as the allocation of subframes, RBs, and power, which we consider in this paper. Other queue-length based scheduling strategies can be found in [22][24]. However, the UE is allowed simultaneous reception from multiple RNs in [22], which is not supported in the LTE-Advanced Standard; [23] only focuses on the out-band relaying scheme, without considering the power allocation; In [24], without considering power adaption, equal subframe is allocated to the backhaul link and the access link in the Type 1 inband relaying scheme, leading to a sub-optimal solution.

\section{System Model AND PRoblem Statement}

We consider the downlink transmission in the LTEAdvanced Type 1 relaying network as illustrated in Fig. 1, with one eNB and $K$ RNs in a specific area. We denote the set containing the eNB and RNs as $\mathcal{B}=\{0,1, \cdots, K\}$, where 0 refers to the eNB. We denote the RN set as $\mathcal{R}=\{1, \cdots, K\}$. The important notations used throughout this paper are summarized in Table I.

TABLE I

NOTATION SUMMARY

\begin{tabular}{|c|l|}
\hline $\mathcal{B}$ & eNB and RN set \\
\hline $\mathcal{R}$ & RN set \\
\hline $\mathcal{U}_{k}$ & Set of UEs served by RN $k$ or by the eNB when $k=0$ \\
\hline $\mathcal{J}$ & RB set \\
\hline$s_{A}(t)$ & Allocation of subframe $t$ to the AL \\
\hline$s_{B}(t)$ & Allocation of subframe $t$ to the BL \\
\hline$b_{m k j}(t)$ & $\begin{array}{l}\text { Allocation of RB } j \text { to UE }(m, k) \text { in BL or to UE }(m, 0) \text { in } \\
\text { DL in subframe } t\end{array}$ \\
\hline$a_{m k j}(t)$ & Allocation of RB $j$ to UE $(m, k)$ in AL in subframe $t$ \\
\hline$c_{j}(t)$ & Indicate function for the usage of RB $j$ in subframe $t$ \\
\hline$P_{k}$ & $\begin{array}{l}\text { Average power constraint for RN } k \text { or for the eNB when } \\
k=0\end{array}$ \\
\hline$\hat{P}_{k}$ & $\begin{array}{l}\text { Instantaneous power constraint for RN } k \text { or for the eNB when } \\
k=0\end{array}$ \\
\hline$p_{m k j}^{B}(t)$ & $\begin{array}{l}\text { Transmission power allocated to UE }(m, k) \text { in BL or to the } \\
\text { UE }(m, 0) \text { in DL over RB } k \text { in subframe } t\end{array}$ \\
\hline$p_{m k j}^{A}(t)$ & $\begin{array}{l}\text { Transmission power allocated to UE }(m, k) \text { in AL over RB } \\
j \text { in subframe } t\end{array}$ \\
\hline$p_{k}(t)$ & $\begin{array}{l}\text { Instantaneous power of RN } k \text { or of the eNB when } k=0 \text { in } \\
\text { subframe } t\end{array}$ \\
\hline$g_{m 0 j}^{D}(t)$ & $\begin{array}{l}\text { Channel gain of RB } j \text { between UE }(m, 0) \text { and eNB in DL } \\
\text { in subframe } t\end{array}$ \\
\hline$g_{k 0 j}^{B}(t)$ & $\begin{array}{l}\text { Channel gain of RB } j \text { between RN } k \text { and eNB in BL in } \\
\text { subframe } t\end{array}$ \\
\hline$g_{m k j}^{A}(t)$ & $\begin{array}{l}\text { Channel gain of RB } j \text { between UE }(m, k) \text { and RN } k \text { in AL } \\
\text { in subframe } t\end{array}$ \\
\hline$r_{m k}^{B}(t)$ & $\begin{array}{l}\text { Transmission rate for UE }(m, k) \text { in BL or for UE }(m, 0) \text { in } \\
\text { DL in subframe } t\end{array}$ \\
\hline$r_{m k}^{A}(t)$ & Transmission rate for UE $(m, k)$ in AL in subframe $t$ \\
\hline$Z_{m k}(t)$ & Application layer queue length for UE $(m, k)$ in subframe $t$ \\
\hline$\beta_{m k}(t)$ & $\begin{array}{l}\text { Admitted amount of data for UE }(m, k) \text { from the application } \\
\text { layer queue in subframe } t\end{array}$ \\
\hline$\beta_{m a x}$ & $\left.\begin{array}{l}\text { Upper bound for } \beta m k \\
m\end{array}\right)$ regarding each UE in each subframe \\
\hline$Q_{m k}(t)$ & Queue length at the eNB for UE $(m, k)$ in subframe $t$ \\
\hline$H_{m k}(t)$ & Queue length at RN $k$ for UE $(m, k)$ in subframe $t$ \\
\hline$\Theta_{k}(t)$ & $\begin{array}{l}\text { Virtual queue length for the average power constraint in } \\
\text { subframe } t\end{array}$ \\
\hline$Y_{m k}(t)$ & $\begin{array}{l}\text { Virtual queue length for the auxiliary variable constraint in } \\
\text { subframe } t\end{array}$ \\
\hline
\end{tabular}




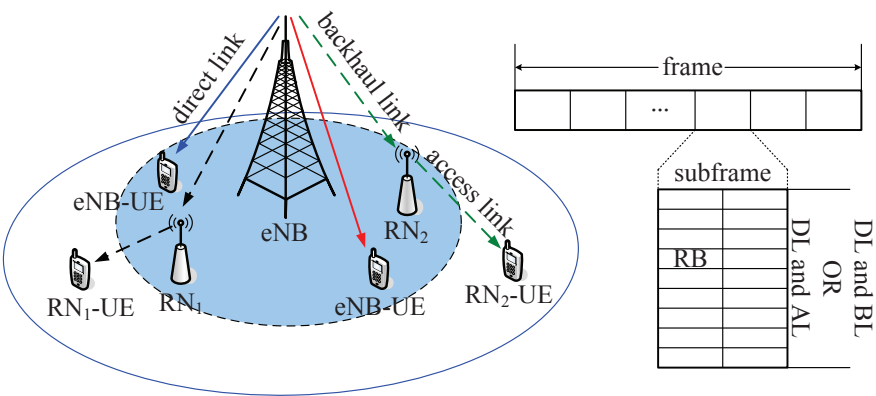

Fig. 1. LTE-Advanced inband relay network and frame architecture

\section{A. LTE-Advanced Type 1 Inband Relaying}

The LTE-Advanced standard does not support simultaneous reception of signals from eNB and RN by an UE. The UE can be served either directly by the eNB or by one RN. The UE closer to the eNB directly connects with the eNB, while the UE that is further from the eNB communicates with its closest $\mathrm{RN}$. We denote the set of UEs served by the eNB as $\mathcal{U}_{0}$ and those served by $\mathrm{RN} k$ as $\mathcal{U}_{k}$ for $k=1,2, \cdots, K$. The number of UEs in the set $\mathcal{U}_{k}$ is $M_{k}=\left|\mathcal{U}_{k}\right|$, so the total number of UEs is expressed as $M=\sum_{k \in \mathcal{B}} M_{k}$ and the number of UEs attached to RNs is $M_{R}=\sum_{k \in \mathcal{R}} M_{k}$. Furthermore, we use indices of the form $(m, k)$ to specify one UE, indicating that the UE $m$ is attached to the eNB when $k=0$ or attached to $\mathrm{RN} k$ when $k \neq 0$. We term the link between eNB and an UE the direct link (DL).

For the reasons explained in Section I, we focus on Type 1 relays in the standard [1]. The backhaul and access links share the same frequency spectrum in Type 1 relays. They operate in the time-division multiplexing mode, such that in each subframe, all RBs are used exclusively either in the direct and backhaul links, or in the direct and access links. We denote the set of RBs as $\mathcal{J}$ and index each RB by $j$. The total number of RBs is $J=|\mathcal{J}|$. We do not consider RB reuse here, i.e., each RB may be assigned to only one UE for the transmission in any link. Hence, for each subframe, the problem for the scheduler is to decide its activation among the three types of links, and to assign RBs and power to UEs.

The above assumption excludes RB reuse between the direct link and the access link. This benefits interference control in the scenario where the eNB and the $\mathrm{RN}$ are near each other. Given the NP hardness of power control in interferencelimited systems [25], in this paper, we aim to provide an efficient and optimal network control algorithm for Type 1 relaying LTE-Advanced systems under the orthogonal RB allocation constraint. In addition, if the eNB and the RN are geographically far enough such that their mutual interference can be ignored, our proposed algorithm can be easily extended without changing its structure, by allowing both the eNB-UE links and the RN-UE links to use all RBs in the access link activation subframe, while guaranteeing the RB orthogonality only within the eNB-UE links and within the RN-UE links.

\section{B. Subframe Allocation}

Since the backhaul and access links of a Type 1 inband RN use the same frequency spectrum, they cannot be simultaneously active in the same subframe. We use binary variables $s_{B}(t)$ and $s_{A}(t)$ as indicate functions for the allocation of subframe $t$ to the backhaul link and the access link, respectively. We have $s_{A}(t)+s_{B}(t) \leq 1, \forall t$. Note that the direct link may use any subframe.

\section{Resource Block Allocation}

In each subframe, either direct link and access link can transmit simultaneously, or direct link and backhaul link can transmit simultaneously, but different RBs must be allocated to these links and to different UEs. We introduce the binary variable $b_{m k j}(t)$ as an indicator function for the allocation of RB $j$ to UE $(m, k)$ in subframe $t$ on the backhaul link, for $k=1,2, \ldots, K$. We similarly define $a_{m k j}(t)$ in subframe $t$ for the access link. We also similarly define $b_{m 0 j}(t)$ in subframe $t$ for the direct link. Then the no-reuse constraint on RBs implies

$$
\begin{aligned}
c_{j}(t) \triangleq & s_{A}(t) \sum_{k \in \mathcal{R}} \sum_{m \in \mathcal{U}_{k}} a_{m k j}(t)+s_{B}(t) \sum_{k \in \mathcal{R}} \sum_{m \in \mathcal{U}_{k}} b_{m k j}(t) \\
& +\sum_{m \in \mathcal{U}_{0}} b_{m 0 j}(t) \leq 1, \quad \forall j \in \mathcal{J} .
\end{aligned}
$$

We note from the above that there is a non-linear relation between the subframe and RB allocation decisions, which brings challenge to joint optimization.

\section{Average and Instantaneous Power Constraints}

Both the eNB and RNs have average and instantaneous power constraints. We denote the average power constraint for the eNB and RNs as $P_{k}$, and the instantaneous power constraint as $\hat{P}_{k}$, for $k=\{0,1, \cdots, K\}$

Let the transmission power allocated to RB $j$ in subframe $t$ be $p_{m 0 j}^{B}(t)$ for the direct link regarding UE $(m, 0)$, and $p_{m k j}^{B}(t), k \neq 0$, for the backhaul link regarding UE $(m, k)$. Then the instantaneous power of the eNB can be written as

$$
\begin{aligned}
p_{0}(t) \triangleq & s_{B}(t) \sum_{j \in \mathcal{J}} \sum_{k \in \mathcal{R}} \sum_{m \in \mathcal{U}_{k}} b_{m k j}(t) p_{m k j}^{B}(t)+ \\
& \sum_{j \in \mathcal{J}} \sum_{m \in \mathcal{U}_{0}} b_{m 0 j}(t) p_{m 0 j}^{B}(t) .
\end{aligned}
$$

It then follows that the instantaneous power constraint for the eNB can be expressed as $p_{0}(t) \leq \hat{P}_{0}$, and the average power constraint for the eNB can be expressed as $\bar{p}_{0} \triangleq$ $\lim _{u \rightarrow \infty} \frac{1}{u} \sum_{t=1}^{u} p_{0}(t) \leq P_{0}$.

We similarly define $p_{m k j}^{A}(t)$ for the access link, so that the instantaneous power of $\mathrm{RN} k$ can be written as

$$
p_{k}(t)=s_{A}(t) \sum_{j \in \mathcal{J}} \sum_{m \in \mathcal{U}_{k}} a_{m k j}(t) p_{m k j}^{A}(t) .
$$

Then the instantaneous power constraint for RN $k$ can be expressed as $p_{k}(t) \leq \hat{P}_{k}$, and the average power constraint for the RN $k$ is written as $\bar{p}_{k} \triangleq \lim _{u \rightarrow \infty} \frac{1}{u} \sum_{t=1}^{u} p_{k}(t) \leq P_{k}$. 
The above formulation corresponds to a fast power control scheme on the subframe basis as specified in the LTEAdvanced standard. Note that a slow power control scheme on the frame basis, where the link adaptation may be achieved through adaptive modulation and coding, can also be adopted in the standard. However, it is outside the scope of this paper.

\section{E. Transmission Rates}

In subframe $t$, let $g_{m 0 j}^{D}(t)$ be the channel gain of $\mathrm{RB} j$ between the eNB and $\operatorname{UE}(m, 0)$ in the direct link, $g_{k 0 j}^{B}(t)$ be the channel gain of RB $j$ between the eNB and $\mathrm{RN} k$ in the backhaul link, and $g_{m k j}^{A}(t)$ be the channel gain of RB $j$ between RN $k$ and UE $(m, k)$ in the access link. We assume that $g_{m 0 j}^{D}(t), g_{k 0 j}^{B}(t)$, and $g_{m k j}^{A}(t)$ are a constant in each subframe but can dynamically change in different subframes. We further suppose that the channel gains are ergodic and follows a certain continuous distribution, but we do not need to know the distribution.

Then the transmission rate for UE $(m, 0)$ in the direct link can be expressed as

$$
r_{m 0}^{B}(t)=\sum_{j \in \mathcal{J}} R_{b} b_{m 0 j}(t) \log \left(1+\frac{g_{m 0 j}^{D}(t) p_{m 0 j}^{B}(t)}{\sigma^{2}}\right),
$$

where $R_{b}$ is the symbol rate and $\sigma^{2}$ is the noise power. We can write the time averaged transmission rate as $\bar{r}_{m 0}^{B} \triangleq$ $\lim _{u \rightarrow \infty} \frac{1}{u} \sum_{t=1}^{u} r_{m 0}^{B}(t)$. Similarly, the transmission rate in the backhaul link for UE $(m, k)$ can be written as

$$
r_{m k}^{B}(t)=s_{B}(t) \sum_{j \in \mathcal{J}} R_{b} b_{m k j}(t) \log \left(1+\frac{g_{k 0 j}^{B}(t) p_{m k j}^{B}(t)}{\sigma^{2}}\right)
$$

Its corresponding time averaged transmission rate is $\bar{r}_{m k}^{B} \triangleq$ $\lim _{u \rightarrow \infty} \frac{1}{u} \sum_{t=1}^{u} r_{m k}^{B}(t)$. The access link transmission rate for the $\operatorname{UE}(m, k)$ can also be written as

$$
r_{m k}^{A}(t)=s_{A}(t) \sum_{j \in \mathcal{J}} R_{b} a_{m k j}(t) \log \left(1+\frac{g_{m k j}^{A}(t) p_{m k j}^{A}(t)}{\sigma^{2}}\right) .
$$

Its corresponding time averaged transmission rate is $\bar{r}_{m k}^{A} \triangleq$ $\lim _{u \rightarrow \infty} \frac{1}{u} \sum_{t=1}^{u} r_{m k}^{A}(t)$. Since the data of a RN assisted UE is transmitted on the backhaul and access links, we need to balance the transmission on both links with dynamic subframe and $\mathrm{RB}$ allocation.

Under an instantaneous power constraint, we further assume that all transmission rates satisfy the following constraints on their second moments:

$$
\begin{aligned}
& \mathbb{E}\left\{r_{m k}^{B}(t)^{2}\right\} \leq r_{\max }^{2}, \forall m \in \mathcal{U}_{k}, k \in \mathcal{B}, \\
& \mathbb{E}\left\{r_{m k}^{A}(t)^{2}\right\} \leq r_{\max }^{2}, \forall m \in \mathcal{U}_{k}, k \in \mathcal{R},
\end{aligned}
$$

where $r_{\max }^{2}$ is a positive constant. Most widely used channel models, e.g., Rayleigh fading, satisfy the above requirement.

\section{F. Traffic Arrival Process and Queue Updating Functions}

We denote $\eta_{m k}(t)$ as the amount of data arriving at the application layer of the eNB destined for $\operatorname{UE}(m, k)$ in subframe $t$. The data is first injected into an outgoing queue in the application layer, with queue length $Z_{m k}(t)$ in subframe $t$. In the case of finite capacity, the overflowed data will be dropped. An admission control scheme will then determine the admitted amount of data for each $\operatorname{UE}(m, k)$ in each subframe $t$, which is denoted as $\beta_{m k}(t)$.

In addition, separate transmission queues are maintained at the eNB and RN $k$ for each UE $(m, k)$, and the queue lengths at the eNB and RN $k$ in subframe $t$ is denoted as $Q_{m k}(t)$ and $H_{m k}(t)$, respectively. The admitted data from the outgoing queue in the application layer are then injected into the queue at the eNB. To avoid queue instability due to an infinity input, we enforce an upper bound, $\beta_{\max }$, on the admitted data for each UE in each subframe $t$.

Since the admitted data cannot be more than the data in the application layer outgoing queue in subframe $t$, we in addition have $\beta_{m k}(t) \leq Z_{m k}(t)$. Then, the time averaged throughput for $\operatorname{UE}(m, k)$ is expressed as $\bar{\beta}_{m k} \triangleq$ $\lim _{u \rightarrow \infty} \frac{1}{u} \sum_{t=1}^{u} \beta_{m k}(t)$

Based on the above, we can express the queue updating functions for $\mathrm{UE}(m, k)$ as

$$
\begin{aligned}
Z_{m k}(t+1) & =Z_{m k}(t)-\beta_{m k}(t)+\eta_{m k}(t), \\
Q_{m k}(t+1) & =\max \left\{Q_{m k}(t)-r_{m k}^{B}(t), 0\right\}+\beta_{m k}(t),
\end{aligned}
$$

and

$$
H_{m k}(t+1)=\max \left\{H_{m k}(t)-r_{m k}^{A}(t), 0\right\}+r_{m k}^{B}(t) .
$$

\section{G. Dynamic Joint Resource Optimization Problem}

Our objective of joint resource allocation is to maximize the total UE utility, which is a function of the average throughput for each UE. In other words, we want to maximize $\sum_{k \in \mathcal{B}} \sum_{m \in \mathcal{U}_{k}} U\left(\bar{\beta}_{m k}\right)$, where $U(\cdot)$ is some concave nondecreasing function. A typical example of $U(\cdot)$ is $\log (\cdot)$, through maximizing which we can maintain proportional fairness among UEs. Then the optimization problem can be expressed as follows:

$$
\max _{\left\{\boldsymbol{\beta}, \mathbf{s}, \mathbf{a}, \mathbf{b}, \mathbf{p}^{A}, \mathbf{p}^{B}\right\}}\left\{\sum_{k \in \mathcal{B}} \sum_{m \in \mathcal{U}_{k}} U\left(\bar{\beta}_{m k}\right)\right\}
$$

s.t.

$$
\begin{array}{ll}
\bar{\beta}_{m k}<\bar{r}_{m k}^{B}, & \forall m \in \mathcal{U}_{k}, k \in \mathcal{B}, \\
\bar{r}_{m k}^{B}<\bar{r}_{m k}^{A}, & \forall m \in \mathcal{U}_{k}, k \in \mathcal{R}, \\
\bar{p}_{k} \leq P_{k}, & \forall k \in \mathcal{B}, \\
\beta_{m k}(t) \leq Z_{m k}(t), & \forall m \in \mathcal{U}_{k}, k \in \mathcal{B}, t, \\
\beta_{m k}(t) \leq \beta_{\max }, & \forall m \in \mathcal{U}_{k}, k \in \mathcal{B}, t, \\
p_{k}(t) \leq \hat{P}_{k}, & \forall k \in \mathcal{B}, t, \\
c_{j}(t) \leq 1, & \forall j \in \mathcal{J}, t, \\
b_{m k j}(t) \in\{0,1\}, & \forall m \in \mathcal{U}_{k}, k \in \mathcal{B}, t, \\
a_{m k j}(t) \in\{0,1\}, & \forall m \in \mathcal{U}_{k}, k \in \mathcal{R}, t, \\
s_{A}(t)+s_{B}(t) \leq 1, & \forall t, \\
s_{A}(t) \in\{0,1\}, & \forall t, \\
s_{B}(t) \in\{0,1\}, & \forall t,
\end{array}
$$

where $\boldsymbol{\beta}=\left[\beta_{m k}(t)\right]_{1 \times M}, \mathbf{s}=\left[s_{A}(t), s_{B}(t)\right], \mathbf{a}=$ $\left[a_{m k j}(t)\right]_{M_{R} \times J}, \mathbf{b}=\left[b_{m k j}(t)\right]_{M \times J}, \mathbf{p}^{A}=\left[p_{m k j}^{A}(t)\right]_{M_{R} \times J}$, 
and $\mathbf{p}^{B}=\left[p_{m k j}^{B}(t)\right]_{M \times J}$. We denote its optimum as $\sum_{k \in \mathcal{B}} \sum_{m \in \mathcal{U}_{k}} U\left(\bar{\beta}_{m k}^{\mathrm{opt}}\right)$.

The constraints (13) and (14) are to ensure stability of the eNB queues and RN queues, respectively. Equations (15) and (18) are the average and instantaneous power constraints for the eNB and RNs. The constraint (16) ensures that the admitted data cannot be more than the data in the outgoing queue. The constraint (19) ensures that each RB can only be assigned to one UE. And the constraint (22) guarantees that the backhaul and access links cannot simultaneously transmit in the same subframe. It should be noted that $\mathbf{a}, \mathbf{b}$, and $\mathbf{s}$ are all binary variables, which increases the complexity of the problem.

\section{Optimal Dynamic Joint Resource SCHEDUling STRATEGY}

In this section, we present an optimal admission control and dynamic joint subframe, RB, and power allocation strategy (JFRP) for the optimization problem (12)-(24). Its outline is based on a general Lyapunov optimization approach [4].

Since the objective function (12) is a function of the time averaged throughput, to transfer it to an optimization problem with a time averaged objective function, we adopt the standard approach of introducing an auxiliary variable $\alpha_{m k}(t)$ for each UE $(m, k)$ which satisfies $\alpha_{m k}(t) \leq \beta_{\text {max }}$. We denote $\bar{\alpha}_{m k} \triangleq \lim _{u \rightarrow \infty} \frac{1}{u} \sum_{t=1}^{u} \alpha_{m k}(t)$. Then the above optimization is transferred to the following equivalent problem [4]:

$$
\max _{\left\{\boldsymbol{\alpha}, \boldsymbol{\beta}, \mathbf{s}, \mathbf{a}, \mathbf{b}, \mathbf{p}^{A}, \mathbf{p}^{B}\right\}} \sum_{k \in \mathcal{B}} \sum_{m \in \mathcal{U}_{k}} \overline{U\left(\alpha_{m k}\right)}
$$

s.t.

$$
\begin{array}{ll}
\bar{\alpha}_{m k} \leq \bar{\beta}_{m k}, & \forall m \in \mathcal{U}_{k}, k \in \mathcal{B}, \\
\alpha_{m k}(t) \leq \beta_{\max }, & \forall m \in \mathcal{U}_{k}, k \in \mathcal{B}, t, \\
(13)-(24), &
\end{array}
$$

where $\overline{U\left(\alpha_{m k}\right)}=\lim _{u \rightarrow \infty} \frac{1}{u} \sum_{t=1}^{u} U\left(\alpha_{m k}(t)\right)$, and $\boldsymbol{\alpha}=$ $\left[\alpha_{m k}(t)\right]_{1 \times M}$.

\section{A. General Lyapunov Optimization Approach}

To ensure the average power constraint (15), we construct a virtual power queue for each of the eNB and RNs, whose queue length in subframe $t$ is denoted as $\Theta_{k}(t)$, and whose updating function is

$$
\Theta_{k}(t+1)=\max \left\{\Theta_{k}(t)-P_{k}, 0\right\}+p_{k}(t) .
$$

We also construct a virtual queue to satisfy each auxiliary constraint (26), whose queue length in subframe $t$ is denoted as $Y_{m k}(t)$ for the $\mathrm{UE}(m, k)$, and whose updating function is

$$
Y_{m k}(t+1)=\max \left\{Y_{m k}(t)-\beta_{m k}(t), 0\right\}+\alpha_{m k}(t) .
$$

For simple notation, let us denote

$$
\Omega(t)=\left\{Q_{m k}(t), H_{m k}(t), \Theta_{k}(t), Y_{m k}(t)\right\} .
$$

In addition, we define the Lyapunov function as

$$
\begin{aligned}
L(\Omega(t))= & \frac{1}{2} \mathbb{E}\left\{\sum_{k \in \mathcal{B}} \sum_{m \in \mathcal{U}_{k}} Q_{m k}(t)^{2}+\sum_{k \in \mathcal{R}} \sum_{m \in \mathcal{U}_{k}} H_{m k}(t)^{2}+\right. \\
& \left.\sum_{k \in \mathcal{B}} \sum_{m \in \mathcal{U}_{k}} Y_{m k}(t)^{2}+\sum_{k \in \mathcal{B}} \Theta_{k}(t)^{2}\right\} .
\end{aligned}
$$

Then the Lyapunov conditional drift-plus-penalty function can be written as

$$
\begin{aligned}
& \Delta(\Omega(t))= \\
& \mathbb{E}\left\{L(\Omega(t+1))-L(\Omega(t))-V \sum_{k \in \mathcal{B}} \sum_{m \in \mathcal{U}_{k}} U\left(\alpha_{m k}(t)\right) \mid \Omega(t)\right\} \\
& \leq C+\sum_{k \in \mathcal{B}} \sum_{m \in \mathcal{U}_{k}}\left\{\left(Q_{m k}(t)-Y_{m k}(t)\right) \beta_{m k}(t)+\right. \\
& \left.Y_{m k}(t) \alpha_{m k}(t)-V U\left(\alpha_{m k}(t)\right)\right\}-\sum_{m \in \mathcal{U}_{0}} Q_{m 0}(t) r_{m 0}^{B}(t)- \\
& \sum_{k \in \mathcal{R}} \sum_{m \in \mathcal{U}_{k}}\left\{\left(Q_{m k}(t)-H_{m k}(t)\right) r_{m k}^{B}(t)+H_{m k}(t) r_{m k}^{A}(t)\right\} \\
& +\sum_{k \in \mathcal{B}}\left(\Theta_{k}(t) p_{k}(t)-\Theta_{k}(t) P_{k}\right),
\end{aligned}
$$

where

$$
\begin{aligned}
C= & \frac{1}{2} \mathbb{E}\left\{\sum_{k \in \mathcal{B}} \sum_{m \in \mathcal{U}_{k}}\left(r_{m k}^{B}(t)^{2}+\alpha_{m k}(t)^{2}+2 \beta_{m k}(t)^{2}\right)+\right. \\
& \left.\sum_{k \in \mathcal{R}} \sum_{m \in \mathcal{U}_{k}}\left(r_{m k}^{B}(t)^{2}+r_{m k}^{A}(t)^{2}\right)+\sum_{k \in \mathcal{B}}\left(P_{k}^{2}+p_{k}(t)^{2}\right)\right\} \\
< & \frac{3}{2} M\left(r_{\max }^{2}+\beta_{\max }^{2}\right)+\frac{1}{2} \sum_{k \in \mathcal{B}}\left\{P_{k}^{2}+\hat{P}_{k}^{2}\right\} .
\end{aligned}
$$

Following the general Lyapunov optimization approach [4], our dynamic joint resource optimization strategy for the LTEAdvanced Type 1 inband relay network is based on minimizing the RHS of (33). This minimization is non-trivial, however, mainly due to mixed-integer nature of (33). Next, we decompose this problem into three sub-problems and provide an optimal solution to each.

\section{B. Optimal Decision for the Auxiliary Variables}

The optimal decision for the auxiliary variables on the RHS of (33) is made based on minimizing $\sum_{k \in \mathcal{B}} \sum_{m \in \mathcal{U}_{k}}\left(Y_{m k}(t) \alpha_{m k}(t)-V U\left(\alpha_{m k}(t)\right)\right)$, so we can determine the optimal auxiliary variable for each UE separately. We write the optimization problem for $\operatorname{UE}(m, k)$ as

$$
\begin{array}{ll} 
& \min _{\alpha_{m k}(t)} Y_{m k}(t) \alpha_{m k}(t)-V U\left(\alpha_{m k}(t)\right) \\
\text { s.t. } & 0 \leq \alpha_{m k}(t) \leq \beta_{\max } .
\end{array}
$$

It is a convex optimization problem since the objective function is the summation of a linear function $Y_{m k}(t) \alpha_{m k}(t)$ and a convex function $-V U\left(\alpha_{m k}(t)\right)$. By differentiating, the optimal solution can be easily derived. In a special case of $U(\cdot)=\log (\cdot)$, we can obtain the optimal solution as $\alpha_{m k}^{\mathrm{JFRP}}(t)=\min \left\{\frac{V}{Y_{m k}(t)}, \beta_{\max }\right\}$. Clearly, a larger $Y_{m k}(t)$ will 
decrease $\alpha_{m k}^{\mathrm{JFRP}}(t)$ in the current subframe $t$, which in turn avoids the further increase of $Y_{m k}(t)$.

\section{Optimal Admission Control}

To minimize the RHS of (33), we also need to minimize $\sum_{k \in \mathcal{B}} \sum_{m \in \mathcal{U}_{k}}\left(Q_{m k}(t)-Y_{m k}(t)\right) \beta_{m k}(t)$, which may be viewed as an optimal admission control problem. Clearly, we may consider each UE separately. The optimization problem for $\mathrm{UE}(m, k)$ in each subframe $t$ can be expressed as follows:

$$
\begin{aligned}
& \min _{\beta_{m k}(t)}\left(Q_{m k}(t)-Y_{m k}(t)\right) \beta_{m k}(t) \\
\text { s.t. } & \beta_{m k}(t) \leq Z_{m k}(t), \\
& \beta_{m k}(t) \leq \beta_{\max } .
\end{aligned}
$$

This is a linear problem with the following solution:

$\beta_{m k}^{\mathrm{JFRP}}(t)= \begin{cases}\min \left\{Z_{m k}(t), \beta_{\max }\right\}, & \text { if } Q_{m k}(t)-Y_{m k}(t)<0 \\ 0, & \text { otherwise }\end{cases}$

\section{Optimal Subframe, RB, and Power Allocation}

The final, and most challenging, task is to minimize the remaining terms in the RHS of (33). This involves joint allocation of subframes, RBs, and power. We express the optimization problem as follows:

$$
\begin{gathered}
\min _{\left\{\mathbf{s}, \mathbf{a}, \mathbf{b}, \mathbf{p}^{A}, \mathbf{p}^{B}\right\}}\left\{-\sum_{m \in \mathcal{U}_{0}} Q_{m 0}(t) r_{m, 0}^{B}(t)+\sum_{k \in \mathcal{B}} \Theta_{k}(t) p_{k}(t)\right. \\
\left.-\sum_{k \in \mathcal{R}} \sum_{m \in \mathcal{U}_{k}}\left\{\left(Q_{m k}(t)-H_{m k}(t)\right) r_{m k}^{B}(t)+H_{m k}(t) r_{m k}^{A}(t)\right\}\right\}
\end{gathered}
$$

s.t. $(18)-(24)$.

The above objective function is weighted by the virtual power queue lengths and the packet queue lengths. This reflects a balance between the average power constraint and the transmission rate requirement. Note that $r_{m k}^{A}(t), r_{m k}^{B}(t)$, and $p_{k}(t)$ are functions of the subframe allocation binary variable, $s_{A}(t)$ and $s_{B}(t)$, and the $\mathrm{RB}$ allocation binary variables, $a_{m k j}(t)$ and $b_{m k j}(t)$. Combining this with the power allocation, this is a mixed-integer problem, which usually is prohibitively hard to solve. Moreover, the relation between the subframe allocation variables and the RB allocation variables is multiplicative, which further adds to the problem complexity.

However, we show that in the case of (38) the problem can be exactly and efficiently solved through continuity relaxation and Lagrange dual decomposition. Our proposed solution consists of the following four steps:

1) Removal of Binary Multiplication: To make the this problem tractable, we first remove the multiplicative binary variables by introducing auxiliary variables $x_{m k j}=$ $s_{A}(t) a_{m k j}(t)$ and $y_{m k j}=s_{B}(t) b_{m k j}(t), \forall k \in \mathcal{R}$. Clearly, $x_{m k j} \in\{0,1\}$ and $y_{m k j} \in\{0,1\}$. After substituing $x_{m k j}$ and $y_{m k j}$ into $p(t), r_{m k}^{A}(t), r_{m k}^{B}(t)$, and $c_{j}(t)$, we can rewrite the optimization problem (38) as

$$
\min _{\left\{\mathbf{s}, \mathbf{x}, \mathbf{y}, \mathbf{b}_{0}, \mathbf{p}^{A}, \mathbf{p}^{B}\right\}}\left\{-\sum_{m \in \mathcal{U}_{0}} Q_{m 0}(t) r_{m 0}^{B}(t)+\sum_{k \in \mathcal{B}} \Theta_{k}(t) \tilde{p}_{k}(t)\right.
$$

$$
\left.-\sum_{k \in \mathcal{R}} \sum_{m \in \mathcal{U}_{k}}\left\{\left(Q_{m k}(t)-H_{m k}(t)\right) \tilde{r}_{m k}^{B}(t)+H_{m, k}(t) \tilde{r}_{m, k}^{A}(t)\right\}\right\}
$$

s.t.

$$
\begin{aligned}
& \tilde{p}_{k}(t) \leq \hat{P}_{k}, \\
& \tilde{c}_{j}(t) \leq 1, \\
& s_{A}(t)+s_{B}(t) \leq 1, \\
& s_{A}(t) \in\{0,1\}, \\
& s_{B}(t) \in\{0,1\}, \\
& x_{m k j} \in\{0,1\}, \\
& b_{m 0 j}(t) \in\{0,1\}, \\
& y_{m k j} \in\{0,1\},
\end{aligned}
$$

where $\mathbf{x}=\left[x_{m k j}\right]_{M_{R} \times J}, \mathbf{y}=\left[y_{m k j}\right]_{M_{R} \times J}, \mathbf{b}_{0}=$ $\left[b_{m 0 j}(t)\right]_{M_{0} \times J}$,

$\tilde{r}_{m k}^{B}(t)=\sum_{j \in \mathcal{J}} R_{b} y_{m k j} \log \left(1+\frac{g_{k 0 j}^{B}(t) p_{m k j}^{B}(t)}{\sigma^{2}}\right), \forall k \in \mathcal{R}$,

$\tilde{r}_{m k}^{A}(t)=\sum_{j \in \mathcal{J}} R_{b} x_{m k j} \log \left(1+\frac{g_{m k j}^{A}(t) p_{m k j}^{A}(t)}{\sigma^{2}}\right)$,

$\tilde{p}_{0}(t)=\sum_{j \in \mathcal{J}} \sum_{k \in \mathcal{R}} \sum_{m \in \mathcal{U}_{k}} y_{m k j} p_{m k j}^{B}(t)+\sum_{j \in \mathcal{J}} \sum_{m \in \mathcal{U}_{0}} b_{m 0 j}(t) p_{m 0 j}^{B}(t)$,

$\tilde{p}_{k}(t)=\sum_{j \in \mathcal{J}} \sum_{m \in \mathcal{U}_{k}} x_{m k j} p_{m k j}^{A}(t), \forall k \in \mathcal{R}$,

and

$$
\tilde{c}_{j}(t)=\sum_{k \in \mathcal{R}} \sum_{m \in \mathcal{U}_{k}} x_{m k j}+\sum_{k \in \mathcal{R}} \sum_{m \in \mathcal{U}_{k}} y_{m k j}+\sum_{m \in \mathcal{U}_{0}} b_{m 0 j}(t) .
$$

Note that $Q_{m k}(t)-H_{m k}(t)$ is the queue difference between the eNB and RN $k$ with respect to $\mathrm{UE}(m, k)$. It can be less than zero for some $\operatorname{UE}(m, k)$, and in such a case, any RB allocation to the UE will make the term $\Theta_{k}(t) x_{m k j} p_{m k j}^{A}(t)-$ $\left(Q_{m k}(t)-H_{m k}(t)\right) R_{b} x_{m k j} \log \left(1+\frac{g_{m k j}^{A}(t) p_{m k j}^{A}(t)}{\sigma^{2}}\right)>0$. Therefore, no RB will be allocated to such UE. Intuitively, by stopping the transmission in the backhaul link, the queue in the RN will be decreased, and this help balance the transmission between the backhaul link and access link. Hence, we will only focus on the UEs with $Q_{m k}(t)-H_{m k}(t)>0$. Without loss of generality, we assume that all $Q_{m k}(t)-H_{m k}(t)$ are positive in the rest of this section.

2) Continuity Relaxation and Convexification: The above optimization problem is still a mixed-integer nonlinear programming problem, which is typically computational intractable. Here we propose a novel algorithm that can efficiently optimize the joint allocation of subframes, RBs, and power.

To derive the solution to (39)-(47), we introduce auxiliary variables $h_{m k j}=y_{m k j} p_{m k j}^{B}(t), \forall k \in \mathcal{R}, w_{m 0 j}=$ $b_{m 0 j}(t) p_{m 0 j}^{B}(t)$, and $q_{m k j}=x_{m k j} p_{m k j}^{A}(t)$. In addition, we relax the binary variables $b_{m 0 j}(t), s_{A}(t)$, and $s_{B}(t)$ to take value continuously in $[0,1]$. Since $x_{m k j}=s_{A}(t) a_{m k j}(t)$ 
and $y_{m k j}=s_{B}(t) b_{m k j}(t)$, we then have $x_{m k j} \in[0,1]$ and $y_{m k j} \in[0,1]$. Then we can rewrite (39)-(47) as

$$
\begin{aligned}
& \min _{\left\{\mathbf{s}, \mathbf{x}, \mathbf{y}, \mathbf{b}_{0}, \mathbf{h}, \mathbf{q}, \mathbf{w}\right\}}\left\{\sum _ { j \in \mathcal { J } } \sum _ { m \in \mathcal { U } _ { 0 } } \left\{\Theta_{0}(t) w_{m 0 j}-\right.\right. \\
& \left.Q_{m 0}(t) R_{b} b_{m 0 j}(t) \log \left(1+\frac{g_{m 0 j}^{D}(t) w_{m 0 j}}{b_{m 0 j}(t) \sigma^{2}}\right)\right\} \\
& \sum_{j \in \mathcal{J}} \sum_{k \in \mathcal{R}} \sum_{m \in \mathcal{U}_{k}}\left\{\Theta_{0}(t) h_{m k j}+\Theta_{k}(t) q_{m k j}-\right. \\
& \left(Q_{m k}(t)-H_{m k}(t)\right) R_{b} y_{m k j} \log \left(1+\frac{g_{k 0 j}^{B}(t) h_{m k j}}{y_{m k j} \sigma^{2}}\right)- \\
& \left.\left.H_{m k}(t) R_{b} x_{m n j} \log \left(1+\frac{g_{m k j}^{A}(t) q_{m k j}}{x_{m k j} \sigma^{2}}\right)\right\}\right\} \\
& \text { s.t. } \\
& \sum_{j \in \mathcal{J}} \sum_{m \in \mathcal{U}_{0}} w_{m 0 j}+\sum_{j \in \mathcal{J}} \sum_{k \in \mathcal{R}} \sum_{m \in \mathcal{U}_{k}} h_{m k j} \leq \hat{P}_{0} \\
& \sum_{j \in \mathcal{J}} \sum_{m \in \mathcal{U}_{k}} q_{m k j} \leq \hat{P}_{k}, \forall k \in \mathcal{R} \\
& \sum_{k \in \mathcal{R}} \sum_{m \in \mathcal{U}_{k}}\left(x_{m k j}+y_{m k j}\right)+\sum_{m \in \mathcal{U}_{0}} b_{m 0 j}(t) \leq 1, \forall j \in \mathcal{J} \\
& x_{m k j} \in[0,1], y_{m 0 j} \in[0,1], b_{m 0 j}(t) \in[0,1] \\
& s_{A}(t)+s_{B}(t) \leq 1, \\
& s_{A}(t) \in[0,1], s_{B}(t) \in[0,1] \\
& h_{m 0 j} \geq 0, q_{m k j} \geq 0, w_{m k j} \geq 0 \\
& \sum_{m}
\end{aligned}
$$

where $\mathbf{h}=\left[h_{m k j}\right]_{M_{R} \times J}, \mathbf{q}=\left[q_{m k j}\right]_{M_{R} \times J}$, and $\mathbf{w}=$ $\left[w_{m 0 j}\right]_{M_{0} \times J}$.

Note that the term $-Q_{m 0}(t) R_{b} b_{m 0 j}(t) \log \left(1+\frac{g_{m 0 j}^{D}(t) w_{m 0 j}}{b_{m 0 j}(t) \sigma^{2}}\right)$ is the perspective function of the convex function $-Q_{m 0}(t) R_{b} \log \left(1+\frac{g_{m 0 j}^{D}(t) w_{m 0 j}}{\sigma^{2}}\right)$ given that $Q_{m 0}(t) \geq 0$ always holds. Therefore, $-Q_{m 0}(t) R_{b} b_{m 0 j}(t) \log (1+$ $\left.\frac{g_{m 0 j}^{D}(t) w_{m 0 j}}{b_{m 0 j}(t) \sigma^{2}}\right)$ is also a convex function. Using a similar conclusion for the other term in (53), we observe that (53) is a convex function. In addition, since all constraints are linear, the above optimization problem is a convex optimization problem, and the Slater's condition is satisfied. Thus a zero Lagrange duality gap is guaranteed [26].

3) Dual Decomposition: We relax the constraints (54) and (55) by introducing the dual variables $\lambda$ and $\mu_{k}$. The Lagrangian can be written as

$$
\begin{aligned}
& F(\lambda, \boldsymbol{\mu})=(53)+\sum_{k \in \mathcal{R}} \mu_{k}\left(\sum_{j \in \mathcal{J}} \sum_{m \in \mathcal{U}_{k}} q_{m k j}-\hat{P}_{k}\right)+ \\
& \lambda\left(\sum_{j \in \mathcal{J}} \sum_{m \in \mathcal{U}_{0}} w_{m 0 j}+\sum_{j \in \mathcal{J}} \sum_{k \in \mathcal{R}} \sum_{m \in \mathcal{U}_{k}} h_{m k j}-\hat{P}_{0}\right),
\end{aligned}
$$

where $\boldsymbol{\mu}=\left[\mu_{k}\right]_{1 \times K}$. Then the dual function can be derived through

$$
\begin{aligned}
& \qquad D(\lambda, \boldsymbol{\mu})=\min _{\left\{\mathbf{b}_{0}, \mathbf{x}, \mathbf{y}, \mathbf{s}\right\}} F(\lambda, \boldsymbol{\mu}) \\
& \text { s.t. }(56)-(60) \text {. }
\end{aligned}
$$

To derive an optimal solution to the above optimization problem, we write part of its KKT conditions as follows:

$$
\begin{aligned}
& \Theta_{0}(t)-\frac{Q_{m 0}(t) R_{b} b_{m 0 j}(t) g_{m 0 j}^{D}(t)}{\left(g_{m 0 j}^{D}(t) w_{m 0 j}+b_{m 0 j}(t) \sigma^{2}\right) \ln 2}+\lambda+\xi_{m 0 j}=0, \\
& \Theta_{0}(t)-\frac{\left(Q_{m k}(t)-H_{m k}(t)\right) R_{b} y_{m k j} g_{k 0 j}^{B}(t)}{\left(g_{k 0 j}^{B}(t) h_{m k j}+y_{m k j} \sigma^{2}\right) \ln 2}+\lambda+\varphi_{m k j}=0,
\end{aligned}
$$

$\Theta_{k}(t)-\frac{H_{m k}(t) R_{b} x_{m k j} g_{m k j}^{A}(t)}{\left(g_{m k j}^{A}(t) q_{m k j}+x_{m k j} \sigma^{2}\right) \ln 2}+\mu_{k}+\theta_{m k j}=0$,

$\xi_{m 0 j} w_{m 0 j}=0$,

$\varphi_{m k j} h_{m k j}=0$,

$\theta_{m k j} q_{m k j}=0$,

$w_{m 0 j} \geq 0, \quad h_{l 0 j} \geq 0, \quad q_{l n j} \geq 0$,

$\varphi_{m k j} \geq 0, \quad \theta_{m k j} \geq 0, \quad \xi_{m 0 j} \geq 0$.

From (63), we can write

$$
w_{m 0 j}=\left(\frac{Q_{m 0}(t) R_{b}}{\left(\Theta_{0}(t)+\lambda-\xi_{m 0 j}\right) \ln 2}-\frac{\sigma^{2}}{g_{m 0 j}^{D}(t)}\right) b_{m 0 j}(t)
$$

Based on (66), we have that if $w_{m 0 j}>0$, then $\xi_{m 0 j}=0$. Otherwise, if $w_{m 0 j}=0$, then $\xi_{m 0 j} \geq 0$, and it follows that $\frac{Q_{m 0}(t) R_{b}}{\left(\Theta_{0}(t)+\lambda\right) \ln 2} \leq \frac{\sigma^{2}}{g_{m 0 j}^{D}(t)}$. Based on the above, we have

$$
w_{m 0 j}=\left[\frac{Q_{m 0}(t) R_{b}}{\left(\Theta_{0}(t)+\lambda\right) \ln 2}-\frac{\sigma^{2}}{g_{m 0 j}^{D}(t)}\right]^{+} b_{m 0 j}(t)
$$

where $[x]^{+} \triangleq \max \{x, 0\}$. Similarly, we can derive

$$
h_{m k j}=\left[\frac{\left(Q_{m k}(t)-H_{m k}(t)\right) R_{b}}{\left(\Theta_{0}(t)+\lambda\right) \ln 2}-\frac{\sigma^{2}}{g_{k 0 j}^{B}(t)}\right]^{+} y_{m k j},
$$

and

$$
q_{m k j}=\left[\frac{H_{m k}(t) R_{b}}{\left(\Theta_{k}(t)+\mu_{k}\right) \ln 2}-\frac{\sigma^{2}}{g_{m k j}^{A}(t)}\right]^{+} x_{m k j} .
$$

For notation simplicity, we denote

$$
\begin{aligned}
& \Lambda_{m 0 j}(\lambda)=\left[\frac{Q_{m 0}(t) R_{b}}{\left(\Theta_{0}(t)+\lambda\right) \ln 2}-\frac{\sigma^{2}}{g_{m 0 j}^{D}(t)}\right]^{+}, \\
& \Xi_{m k j}(\lambda)=\left[\frac{\left(Q_{m k}(t)-H_{m k}(t)\right) R_{b}}{\left(\Theta_{0}(t)+\lambda\right) \ln 2}-\frac{\sigma^{2}}{g_{k 0 j}^{B}(t)}\right]^{+},
\end{aligned}
$$

and

$$
\Upsilon_{m k j}\left(\mu_{k}\right)=\left[\frac{H_{m k}(t) R_{b}}{\left(\Theta_{k}(t)+\mu_{k}\right) \ln 2}-\frac{\sigma^{2}}{g_{m k j}^{A}(t)}\right]^{+} .
$$


Substituting them into (62) and denoting

$$
\begin{aligned}
\Gamma_{m 0 j}(\lambda)= & \left(\Theta_{0}(t)+\lambda\right) \Lambda_{m 0 j}(\lambda)-Q_{m 0}(t) \\
& R_{b} \log \left(1+\frac{g_{m 0 j}^{D}(t) \Lambda_{m 0 j}(\lambda)}{\sigma^{2}}\right) \\
\Psi_{m k j}(\lambda)= & \left(\Theta_{0}(t)+\lambda\right) \Xi_{m k j}(\lambda)-\left(Q_{m k}(t)-H_{m k}(t)\right) \\
& R_{b} \log \left(1+\frac{\Xi_{m k j}(\lambda) g_{k 0 j}^{B}(t)}{\sigma^{2}}\right)
\end{aligned}
$$

and

$$
\begin{aligned}
\Phi_{m k j}\left(\mu_{k}\right)= & \left(\Theta_{k}(t)+\mu_{k}\right) \Upsilon_{m k j}\left(\mu_{k}\right)-H_{m k}(t) \\
& R_{b} \log \left(1+\frac{g_{m k j}^{A}(t) \Upsilon_{m k j}\left(\mu_{k}\right)}{\sigma^{2}}\right),
\end{aligned}
$$

we can rewrite (62) as

$$
\begin{aligned}
& D(\lambda, \boldsymbol{\mu})=\min _{\left\{\mathbf{b}_{0}, \mathbf{x}, \mathbf{y}\right\}}\left\{\sum_{j \in \mathcal{J}} \sum_{m \in \mathcal{U}_{0}} \Gamma_{m 0 j}(\lambda) b_{m 0 j}(t)+\right. \\
& \left.\sum_{j \in \mathcal{J}} \sum_{k \in \mathcal{R}} \sum_{m \in \mathcal{U}_{k}}\left\{\Psi_{m k j}(\lambda) h_{m k j}+\Phi_{m k j}\left(\mu_{k}\right) q_{m k j}\right\}\right\} \\
& \text { s.t. (56) - (59). }
\end{aligned}
$$

Thus, by using the KKT conditions, we have converted the optimization problem (62) into a linear problem. Combining this with the constraints (56)-(57), the optimal $x_{m k j}^{*}, y_{m k j}^{*}$, and $b_{m 0 j}^{*}(t)$ can only be among the extreme points in the constraint set, i.e., 0 or 1 . Thus, after continuity relaxation on $x_{m k j}$, $y_{m k j}$, and $b_{m 0 j}(t)$, we can still obtain optimal solutions that are binary. The following lemma formalizes this observation.

Lemma 1: The optimal solution, $s_{A}^{*}(t), s_{B}^{*}(t), x_{m k j}^{*}, y_{m k j}^{*}$, and $b_{m 0 j}^{*}(t)$ to (81) satisfies the constraints (41)-(47).

Proof: Note that, in the optimization problem (53)-(60), we have only relaxed $s_{A}(t)$ and $s_{B}(t)$ to be in $[0,1]$. We still have $a_{m k j}(t) \in\{0,1\}$ and $b_{m k j}(t) \in\{0,1\}, k \in \mathcal{R}$. For the optimal solution $x_{m k j}^{*}, y_{m k j}^{*}, b_{m 0 j}^{*}(t)$ to (81), it can be easily verified that $x_{m k j}^{*} \in\{0,1\}, y_{m k j}^{*} \in\{0,1\}$, and $b_{m 0 j}^{*}(t) \in\{0,1\}$ from the constraints (56)-(57). Since $x_{m k j}^{*}=s_{A}^{*}(t) a_{m k j}^{*}(t)$ and $y_{m k j}^{*}=s_{B}^{*}(t) b_{m k j}^{*}(t)$, we have $s_{A}^{*}(t) \in\{0,1\}$ and $s_{B}^{*}(t) \in\{0,1\}$. In addition, we have $s_{A}^{*}(t)+s_{B}^{*}(t) \leq 1$ in (58). It then follows that $s_{A}^{*}(t)$ and $s_{B}^{*}(t)$ cannot both be 1 . Thus, either $s_{A}^{*}(t)=1$ and $s_{B}^{*}(t)=0$, or $s_{A}^{*}(t)=0$ and $s_{B}^{*}(t)=1$. Therefore, the optimal $s_{A}^{*}(t), s_{B}^{*}(t)$, $x_{m k j}^{*}, y_{m k j}^{*}$, and $b_{m 0 j}^{*}(t)$ satisfy the constraints (41)-(47).

Based on Lemma 1, we have either $s_{A}^{*}(t)=1$ and $s_{B}^{*}(t)=$ 0 , or $s_{A}^{*}(t)=0$ and $s_{B}^{*}(t)=1$. We next design a scheme to actually derive $s_{A}^{*}(t), s_{B}^{*}(t), x_{m k j}^{*}, y_{m k j}^{*}$, and $b_{m 0 j}^{*}(t)$. We consider the following two possible actions:

- $s_{A}(t)=1$ and $s_{B}(t)=0$ : Only the direct link and access link transmission is allowed. For each RB $j$, we define a minimum as $W_{j} \triangleq \min \left\{\Gamma_{m 0 j}(\lambda), m \in\right.$ $\left.\mathcal{U}_{0} ; \Phi_{m k j}\left(\mu_{k}\right), k \in \mathcal{R}, m \in \mathcal{U}_{k}\right\}$. If $W_{j}<0$, we set $b_{l 0 j}(t)=1$ for $\operatorname{UE}(l, 0)$ with $\Gamma_{l 0 j}(\lambda)=W_{j}$, and set $x_{l n j}=1$ for $\operatorname{UE}(l, n)$ with $\Phi_{l n j}\left(\mu_{k}\right)=W_{j}$.

- $s_{A}(t)=0$ and $s_{B}(t)=1$ : Only the direct link and backhaul link transmission is allowed. For each RB $j$, we define a minimum as $W_{j} \triangleq \min \left\{\Gamma_{m 0 j}(\lambda), m \in\right.$ $\left.\mathcal{U}_{0} ; \Psi_{m k j}(\lambda), k \in \mathcal{R}, m \in \mathcal{U}_{k}\right\}$. If $W_{j}<0$, we set $b_{l 0 j}(t)=1$ for $\operatorname{UE}(l, 0)$ with $\Gamma_{l 0 j}(\lambda)=W_{j}$, and set $y_{l n j}=1$ for $\operatorname{UE}(l, n)$ with $\Psi_{l n j}(\lambda)=W_{j}$.
We choose the action that gives a smaller $D(\lambda, \boldsymbol{\mu})$ and use the subframe and RB allocation solution therein. It can be easily verified that we can minimize (81) while satisfying the constraints (41)-(47) through the above scheme.

Once the dual function $D(\lambda, \boldsymbol{\mu})$ is derived, the dual optimization problem can be written as

$$
\begin{array}{cl} 
& \max _{\lambda, \boldsymbol{\mu}} D(\lambda, \boldsymbol{\mu}) \\
\text { s.t. } & \lambda \geq 0, \\
& \mu_{k} \geq 0, \forall k \in \mathcal{B} .
\end{array}
$$

Using the subgradient method, we can iteratively compute the optimal solution to the above problem as follows:

$$
\begin{aligned}
& \lambda(\nu+1)=[\lambda(\nu)+ \\
& \left.\epsilon(\nu)\left(\sum_{j \in \mathcal{J}} \sum_{m \in \mathcal{U}_{0}} w_{m 0 j}+\sum_{j \in \mathcal{J}} \sum_{k \in \mathcal{R}} \sum_{m \in \mathcal{U}_{k}} h_{m k j}-\hat{P}_{0}\right)\right]^{+}, \\
& \mu_{k}(\nu+1)=\left[\mu_{k}(\nu)+\epsilon(\nu)\left(\sum_{j \in \mathcal{J}} \sum_{m \in \mathcal{U}_{k}} q_{m k j}-\hat{P}_{k}\right)\right]^{+},
\end{aligned}
$$

where $\nu$ is the iteration index and $\epsilon(\nu)$ is the step size at the $\nu$ th iteration. By choosing a proper sequence for $\epsilon(\nu)$, the optimum of (82) can be derived [27].

4) Primal Recovery: Let $\lambda^{*}$ and $\boldsymbol{\mu}^{*}$ be the optimizers for $(82)$. We then need to recover the optimal solution for the primal problem (53)-(60). However, we observe that the Lagrangian $F(\lambda, \boldsymbol{\mu})$ is not a strictly convex function in terms of $b_{m 0 j}(t), x_{m k j}$, and $y_{m k j}$. Hence, given $\lambda^{*}$ and $\boldsymbol{\mu}^{*}$, we may not uniquely recover the optimal $b_{m 0 j}(t), x_{m k j}$, and $y_{m k j}$. Here we use the allocation of RB $j$ as an example to illustrate this issue. Let $\psi_{j}=\min \left\{\Gamma_{m 0 j}(\lambda), m \in\right.$ $\left.\mathcal{U}_{0} ; \Psi_{m k j}(\lambda), \Phi_{m k j}\left(\mu_{k}\right), k \in \mathcal{R}, m \in \mathcal{U}_{k}\right\}<0$. If more than one $\Gamma_{m 0 j}(\lambda), \Psi_{m k j}(\lambda)$, or $\Phi_{m k j}\left(\mu_{k}\right)$ is equal to $\psi_{j}$, then we cannot decide how to assign RB $j$ among the UEs, since in that case there will be an infinite number of choices for the primal variables to minimize (81), not all of which are optimal or even feasible.

Fortunately, the probability of the above case is zero, as stated in Theorem 1 . Hence, we can almost always uniquely recover the optimal $x_{m k j}^{*}, y_{m k j}^{*}$, and $b_{m 0 j}^{*}(t)$ given $\lambda^{*}$ and $\boldsymbol{\mu}^{*}$.

Theorem 1: Given the optimal $\lambda^{*}$ and $\boldsymbol{\mu}^{*}$, for each $\mathrm{RB} j$, the probability that more than one $\Gamma_{m 0 j}(\lambda), \Psi_{m k j}(\lambda)$, and $\Phi_{m k j}\left(\mu_{k}\right)$ is equal to $\psi_{j}$ in the continuous fading channel model is zero. Thus we can recover the optimal primal variable $b_{m 0 j}^{*}(t), x_{m k j}^{*}$, and $y_{m k j}^{*}$ uniquely with probability 1 .

Proof: Without loss of generality, we only discuss the case that more than one $\Gamma_{m 0 j}(\lambda)$ is equal to $\psi_{j}$ for the RB $j$. A similar conclusion can be applied to the discussion of $\Psi_{m k j}(\lambda), \Phi_{m k j}\left(\mu_{k}\right)$, and the relation among them.

Suppose $\Gamma_{m 0 j}(\lambda)=\psi_{j}$. We inspect the probability that there exist $l \neq m$ such that $\Gamma_{l 0 j}(\lambda)=\psi_{j}$. Since $\psi_{j}<0$, from the expression of $\Gamma_{l 0 j}(\lambda)$, we must have $\Lambda_{l 0 j}(\lambda)>0$. Substituting $\Lambda_{l 0 j}(\lambda)$ into $\Gamma_{l 0 j}(\lambda)$, we can easily see that $\Gamma_{l 0 j}(\lambda)=$ 


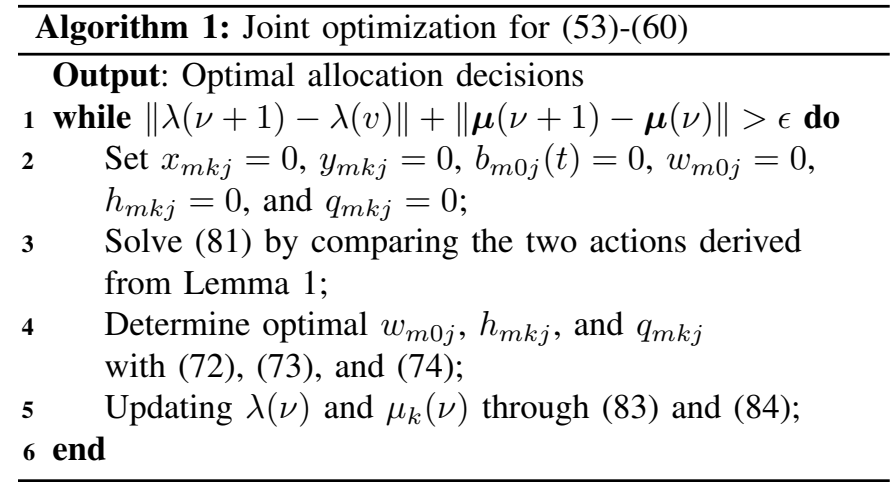

$\frac{Q_{l 0}(t) R_{b}}{\ln 2}-\frac{\left(\Theta_{0}(t)+\lambda\right) \sigma^{2}}{g_{l 0 j}^{D}(t)}-Q_{l 0}(t) R_{b} \log \left(\frac{g_{l 0 j}^{D}(t) Q_{l 0}(t) R_{b}}{\left(\Theta_{0}(t)+\lambda\right) \sigma^{2} \ln 2}\right)$. Now we inspect the relation between $\Gamma_{l 0 j}(\lambda)$ and the channel state $g_{l 0 j}^{D}(t)$. It is obvious that $\Gamma_{l 0 j}(\lambda)$ is a non-flat function regarding $g_{l 0 j}^{D}(t)$, i.e., it does not contain any horizontal line segments. Therefore, there is at most a countable set of values for $g_{l 0 j}^{D}(t)$ to allow $\Gamma_{l 0 j}(\lambda)=\Gamma_{m 0 j}(\lambda)$. Let us denote the set of those points as $\mathcal{N}_{l 0 j}$. Since the continuous fading channel state can take an uncountable number of values, we have $\operatorname{Pr}\left\{g_{l 0 j}^{D}(t) \in \mathcal{N}_{l 0 j}\right\}=0$. Hence, we have $\operatorname{Pr}\left\{\Gamma_{l 0 j}(\lambda)=\Gamma_{m 0 j}(\lambda)=\psi_{j}\right\}=0$.

5) Overall Algorithm and Optimality: In Algorithm 1, we summarize the overall procedure above to solve the optimization problem (53)-(60). We next show how this can directly lead to an optimal solution to (38).

Using Algorithm 1, we can derive the optimal $\mathbf{s}^{*}, \mathbf{x}^{*}, \mathbf{y}^{*}$, $\mathbf{b}_{0}^{*}, \mathbf{q}^{*}, \mathbf{h}^{*}$, and $\mathbf{w}^{*}$ to the continuity relaxed problem (53)-

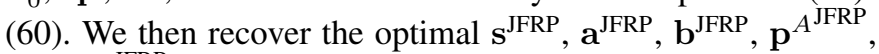
and $\mathbf{p}^{B^{\mathrm{JFRP}}}$ based on the following: $\mathbf{s}^{\mathrm{JFRP}}=\mathbf{s}^{*}, \mathbf{a}^{\mathrm{JFRP}}=\mathbf{x}^{*}$, $\mathbf{b}^{\mathrm{JFRP}}=\mathbf{y}^{*}, \mathbf{b}_{0}^{\mathrm{JFRP}}=\mathbf{b}_{0}^{*}, \mathbf{p}^{A^{\mathrm{JFRP}}}=\mathbf{q}^{*}, \mathbf{p}^{B^{\mathrm{JFRP}}}=\mathbf{h}^{*}$, and $\mathbf{p}_{0}^{B^{\text {JFRP }}}=\mathbf{w}_{0}^{*}$. The reason that we can recover the optimal solution to (38) is that the optimal solution to the continuity relaxed problem (53)-(60) turn out to be binary. This is a main highlight of this algorithm and is summarized in the following theorem.

Theorem 2: With Algorithm 1 and the above recovering scheme, we can derive the optimal subframe, RB, and power allocation, $\mathbf{s}^{\mathrm{JFRP}}, \mathbf{a}^{\mathrm{JFRP}}, \mathbf{b}^{\mathrm{JFRP}}, \mathbf{p}^{A^{\mathrm{JFRP}}}$, and $\mathbf{p}^{B^{\mathrm{JFRP}}}$, to the optimization problem (38).

Proof: From Lemma 1, we know that the optimal solution to (53)-(60) satisfies the constraints (40)-(47). By the above recovering scheme, we can easily derive that the optimal $\mathrm{s}^{\mathrm{JFRP}}$, $\mathbf{a}^{\mathrm{JFRP}}, \mathbf{b}^{\mathrm{JFRP}}, \mathbf{p}^{A^{\mathrm{JFRP}}}$, and $\mathbf{p}^{B^{\mathrm{JFRP}}}$ satisfy the constraints (18)(24). Since the optimization (53)-(60) is a relaxed version of the optimization problem (39)-(47) which is equivalent to the optimization problem (38) by introducing the auxiliary variables, the optimum of (53)-(60) should be no more than that of (38). In addition, the optimal solution to the continuity relaxed problem (53)-(60) satisfy the constraints (18)-(24) with the above recovering scheme. We then have the optimum of (53)-(60) is equal to that of (38).

6) Implementation: We briefly discuss the implementation of Algorithm 1 in an LTE-Advanced Type 1 inband relay network. To reduce system complexity, the LTE-Advanced standard advocates that, instead of centralized control by the eNB, a Type 1 relay could implement its own radio resource management (RRM) for transmissions over the access link. To follow Algorithm 1 under such a condition, the system may adopt an iterative Network Utility Maximization approach. The RN first decides on a suboptimal $a_{m k j}(t)$ with its own RRM. It then sends $a_{m k j}(t)$ and the corresponding $\Phi_{m k j}\left(\mu_{k}\right)$ to the eNB. The eNB is responsible for resolving the conflict among UEs at different links. It sends its optimal control decision to the RN, which is then used by the RN to update its dual valuable $\mu_{k}(\nu)$. Such a procedure requires tight cooperation between the eNB and an RN, which may be supported by a relay-dedicated control channel termed R-PDCCH specified in [28].

\section{Performance Bounds And Numerical EVALUATION}

In this section, we first quantify the performance of our proposed dynamic joint resource optimization algorithm (JFRP), and then evaluate its performance through simulation.

\section{A. Utility and Queue Bounds}

Theorem 3: The proposed dynamic joint subframe, RB, and power allocation strategy provides the following performance guarantees:

$$
\begin{aligned}
& \sum_{k \in \mathcal{B}} \sum_{m \in \mathcal{U}_{k}} U\left(\bar{\beta}_{m k}^{\mathrm{JFRP}}\right) \geq \sum_{k \in \mathcal{B}} \sum_{m \in \mathcal{U}_{k}} U\left(\bar{\beta}_{m k}^{\text {opt }}\right)-\frac{C}{V}-\delta \\
& \lim _{t \rightarrow \infty} \frac{1}{t} \sum_{u=1}^{t} \mathbb{E}\left\{\sum_{m \in \mathcal{U}_{k}}\left(\sum_{k \in \mathcal{B}} Q_{m k}(u)^{\mathrm{JFRP}}+\sum_{k \in \mathcal{R}} H_{m k}(u)^{\mathrm{JFRP}}\right)\right\} \\
& \leq \frac{C}{\delta}+V, \\
& \lim _{t \rightarrow \infty} \frac{1}{t} \mathbb{E}\left\{\Theta_{k}(t)^{\mathrm{JFRP}}\right\}=0, \quad \forall k \in \mathcal{B},
\end{aligned}
$$

where $\sum_{k \in \mathcal{B}} \sum_{m \in \mathcal{U}_{k}} U\left(\bar{\beta}_{m k}^{o p t}\right)$ is the optimal solution to (12)(24), and $\delta$ is an arbitrarily small positive constant.

Proof: The proposed control algorithm minimizes the RHS of (33) via the three sub-problems presented in the last section. Then for the optimization problem (25)-(28), we have

$$
\begin{aligned}
& \sum_{k \in \mathcal{B}} \sum_{m \in \mathcal{U}_{k}} \overline{U\left(\alpha_{m k}^{\mathrm{JFRP}}\right)} \geq \sum_{k \in \mathcal{B}} \overline{U\left(\alpha_{m k}^{\text {opt }}\right)}-\frac{C}{V}-\delta, \\
& \lim _{t \rightarrow \infty} \frac{1}{t} \mathbb{E}\left\{Y_{m k}(t)^{\mathrm{JFRP}}\right\}=0, \quad \forall m \in \mathcal{U}_{k}, k \in \mathcal{B},
\end{aligned}
$$

along with (86) and (87), where $\sum_{k \in \mathcal{B}} \sum_{m \in \mathcal{U}_{k}} \overline{U\left(\alpha_{m, k}^{\text {opt }}\right)}$ is the optimum of (25).

Then, Theorem 3 follows from the general derivation in Chapter 5 of [4]. The details are omitted to avoid redundancy.

Note that (86) implies the queues in the eNB and the RNs are stable and upper bounded by $\frac{C}{\delta}+V$. With a large $V$, we can force $\sum_{k \in \mathcal{B}} \sum_{m \in \mathcal{U}_{k}} U\left(\bar{\beta}_{m k}^{\text {JFRP }}\right)$ arbitrarily close to $\sum_{k \in \mathcal{B}} \sum_{m \in \mathcal{U}_{k}} U\left(\bar{\beta}_{m k}^{o p t}\right)$, but paying a cost of linearly increasing transmission delay. 


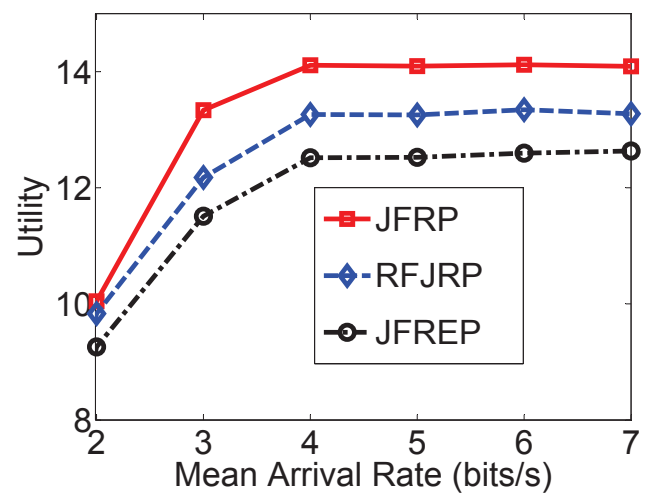

Fig. 2. Sum utility vs. mean arrival rate.

\section{B. Numerical Evaluation}

We then evaluate the performance of the proposed dynamic joint resource allocation algorithm (JFRP) in an example OFDMA based network with Type 1 relaying. The network consists of one eNB and one Type $1 \mathrm{RN}$. The number of eNBUEs and RN-UEs is both set to five, and we specifically take $U(\cdot)=\log (\cdot)$ to maintain proportional fairness among UEs. We model the channel amplitudes as i.i.d. Rayleigh random variables with unit average power for the direct, backhaul, and access links. We normalize the noise power to one and set the average and instantaneous maximal power of the eNB to 20 and 40 . In addition, we set the average and instantaneous maximal power of the $\mathrm{RN}$ to 10 and 20 . The number of RBs $J$ is set to twenty in each subframe, and the maximal admitted data $\beta_{\max }$ is given as seven for each UE. Without loss of generality, we normalize the symbol rate $R_{b}$ to one. The application layer traffic follows the Poisson distribution with a predetermined mean arrival rate.

We compare our algorithm with the Random-subFrameJoint-RB-and-Power allocation (RFJRP) scheme, where subframes for the backhaul link and access link are randomly assigned, and RBs and power are jointly optimized using our algorithm. Note that the RB and power allocation scheme in RFJRP is applicable to the OFDMA based Type 1a outband relay network. Furthermore, we also compare with the JointsubFrame-and-RB-Equal-Power allocation (JFREP) scheme as in [15], where the joint subframe and RB allocation is considered and the maximal power is set to the average power and is equally allocated among the RB.

Setting $V=60$, we first show the throughput utility and the time averaged sum queue length versus the UE traffic mean arrival rate in Fig. 2 and Fig. 3. We observe that the JFRP scheme achieves a higher utility than both RFJRP and JFREP under all arrival rates. Noting that the throughput utility is a $\log$ function, the increase in throughput by JFRP is substantial. We also observe that JFRP achieves smaller queue sizes, and hence lower delay, than RFJRP at all arrival rates and JFREP when the arrival rate is small. Overall, by jointly considering subframe, RB, and power allocation, JFRP can significantly improve over RFJRP in both throughput utility and delay, and over JFREP in throughput utility.

We also study the algorithm performance versus the tuning

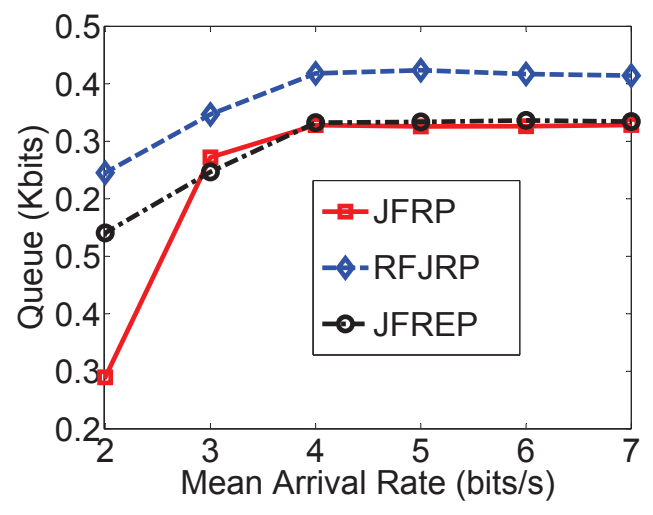

Fig. 3. Sum queue length vs. mean arrival rate.

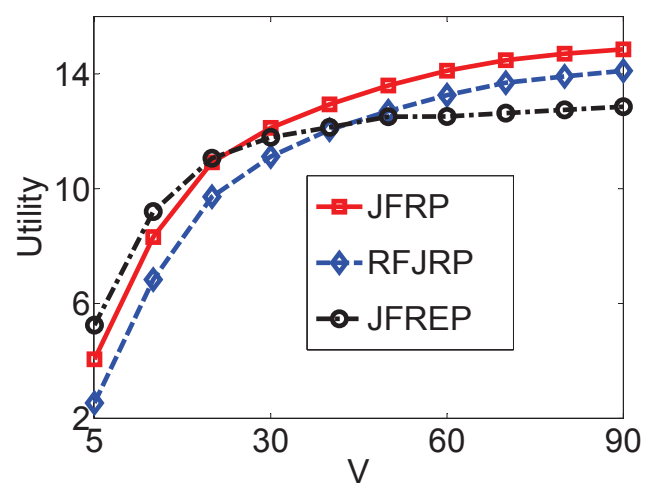

Fig. 4. Sum utility vs. tuning parameter V

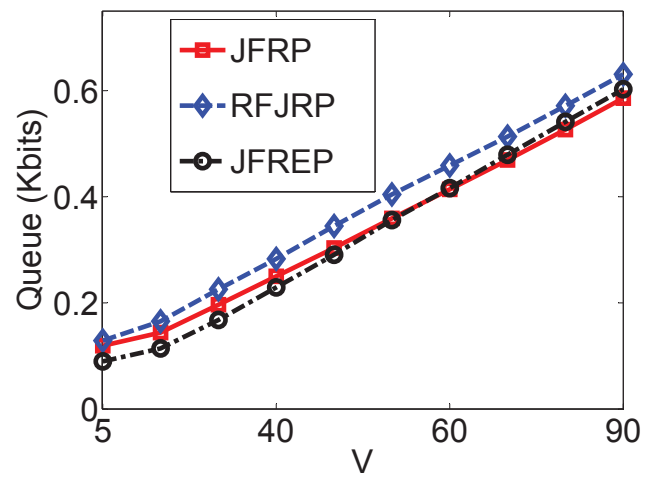

Fig. 5. Sum queue length vs. tuning parameter V

parameter $V$ in Fig. 4 and Fig. 5, where the mean arrival rate is set to 4 bits/slot. We can see that the utility achieved by JFRP outperforms that of RFJRP for all $V$, and it is higher than the utility of JFREP under $V \geq 30$. Note that when $V<30$, the power in JFRP is under utilized, so a proper operating point for JFRP should have $V \geq 30$. We also notice that, with $V>50$, both JFRP and RFJRP outperform JFREP, which implies the importance of the adaptive power allocation over subframe and RB allocation. We can also see from Fig. 5 that the time averaged sum queue length in JFRP is smaller than that of RFJRP and JFREP given $V>50$, which indicates it has a lower delay. 


\section{CONCLUSION}

We study the dynamic joint resource optimization problem in LTE-Advanced networks with Type 1 relaying scheme. To adaptively accommodate the network dynamics, we utilize the general framework of Lyapunov optimization. Our main contribution is in proposing a novel algorithm to minimize the drift-plus-penalty function. Taking advantage of continuity relaxation and Lagrange dual decomposition, the joint subframe, $\mathrm{RB}$, and power allocation problem is efficiently solved. We further quantify the performance of our strategy, showing that a tradeoff between $1-O\left(\frac{1}{V}\right)$ utility and $O(V)$ delay can be achieved, and that the joint optimization can lead to substantial performance improvements over suboptimal alternatives.

\section{REFERENCES}

[1] 3GPP TR 36.814 V9.0.0. (2010) Evolved universal terrestrial radio access (E-UTRA); further advancements for e-utra physical layer aspects. [Online]. Available: http://www.3gpp.org/ftp/Specs/html-info/ 36814.htm

[2] 3GPP TS 36.211 V10.4.0. (2012) Evolved universal terrestrial radio access (E-UTRA);physical channels and modulation. [Online]. Available: http://www.3gpp.org/ftp/Specs/html-info/36211.htm

[3] J. Góra and S. Redana, "Resource management issues for multi-carrier relay-enhanced systems," EURASIP Journal on Wireless Communications and Networking, vol. 2012, pp. 1-18, 2012.

[4] M. Neely, Stochastic network optimization with application to communication and queueing systems. Morgan \& Claypool Publishers, 2010.

[5] K. Sundaresan and S. Rangarajan, "Efficient algorithms for leveraging spatial reuse in OFDMA relay networks," in Proc. IEEE INFOCOM, 2009, pp. 1539-1547.

[6] Z. Yang, Q. Zhang, and Z. Niu, "Throughput improvement by joint relay selection and link scheduling in relay-assisted cellular networks," IEEE Trans. Veh. Technol., no. 99, p. 1, 2012.

[7] M. Hajiaghayi, M. Dong, and B. Liang, "Jointly optimal channel and power assignment for dual-hop multi-channel multi-user relaying," IEEE J. Sel. Areas Commun., October 2012. [Online]. Available: http://arxiv.org/abs/1102.5314

[8] - "Jointly optimal bit loading, channel pairing and power allocation for multi-channel relaying," in Proc. IEEE INFOCOM, 2012, pp. 657 -665 .

[9] Y. Liu and M. Tao, "Optimal channel and relay assignment in OFDMbased multi-relay multi-pair two-way communication networks," IEEE Trans. Commun., vol. 60, no. 2, pp. 317-321, 2012.

[10] Y. Liu, M. Tao, B. Li, and H. Shen, "Optimization framework and graphbased approach for relay-assisted bidirectional ofdma cellular networks," IEEE Trans. Wireless Commun., vol. 9, no. 11, pp. 3490-3500, 2010.

[11] T. C.-Y. Ng and W. Yu, "Joint optimization of relay strategies and resource allocations in cooperative cellular networks," IEEE J. Sel. Areas Commun., vol. 25, no. 2, pp. 328-339, 2007.

[12] K. Sundaresan and S. Rangarajan, "On exploiting diversity and spatial reuse in relay-enabled wireless networks," in Proc. ACM MobiHoc, 2008, pp. 13-22.

[13] Y. Hua, Q. Zhang, and Z. Niu, "Resource allocation in multi-cell OFDMA-based relay networks," in Proc. IEEE INFOCOM, 2010, pp. $1-9$.

[14] M. Kaneko, P. Popovski, and K. Hayashi, "Throughput-guaranteed resource allocation algorithms for relay-aided cellular OFDMA system," IEEE Trans. Veh. Technol., vol. 58, no. 4, pp. 1951-1964, 2009.

[15] K. Sundaresan and S. Rangarajan, "Adaptive resource scheduling in wireless OFDMA relay networks," in Proc. IEEE INFOCOM, 2012, pp. 1080-1088.

[16] Z. Ma, W. Xiang, H. Long, and W. Wang, "Proportional fair resource partition for LTE-advanced networks with Type I relay nodes," in Proc. IEEE ICC, 2011, pp. 1-5.

[17] B. Choi, S. Bae, K. Cheon, A. Park, and M. Chung, "Relay selection and resource allocation schemes for effective utilization of relay zones in relay-based cellular networks," IEEE Commun. Lett., no. 99, pp. 1-3, 2011.

[18] 3GPP TS 36.300 V10.7.0. (2012) Evolved universal terrestrial radio access (E-UTRA) and evolved universal terrestrial radio access network (E-UTRAN); overall description; stage 2. [Online]. Available: http://www.3gpp.org/ftp/Specs/html-info/36300.htm
[19] L. Tassiulas and A. Ephremides, "Stability properties of constrained queueing systems and scheduling policies for maximum throughput in multihop radio networks," IEEE Trans. Autom. Control, vol. 37, no. 12, pp. $1936-1948,1992$.

[20] L. Ying, S. Shakkottai, A. Reddy, and S. Liu, "On combining shortestpath and back-pressure routing over multihop wireless networks," IEEE/ACM Trans. Netw., vol. 19, no. 3, pp. 841 -854, 2011.

[21] M. Neely, E. Modiano, and C.-P. Li, "Fairness and optimal stochastic control for heterogeneous networks," IEEE/ACM Trans. Netw., vol. 16, no. 2, pp. $396-409,2008$.

[22] H. Viswanathan and S. Mukherjee, "Performance of cellular networks with relays and centralized scheduling," IEEE Trans. Wireless Commun., vol. 4, no. 5, pp. 2318-2328, 2005.

[23] M. Salem, A. Adinoyi, M. Rahman, H. Yanikomeroglu, D. Falconer, and Y.-D. Kim, "Fairness-aware radio resource management in downlink ofdma cellular relay networks," IEEE Trans. Wireless Commun., vol. 9, no. 5, pp. 1628-1639, 2010.

[24] M. Salem, A. Adinoyi, H. Yanikomeroglu, and D. Falconer, "Fair resource allocation toward ubiquitous coverage in ofdma-based cellular relay networks with asymmetric traffic," IEEE Trans. Veh. Technol., vol. 60, no. 5, pp. 2280-2292, 2011.

[25] Z.-Q. Luo and S. Zhang, "Dynamic spectrum management: Complexity and duality," IEEE J. Sel. Topics Signal Process., vol. 2, no. 1, pp. 5773, 2008.

[26] S. Boyd and L. Vandenberghe, Convex optimization. Cambridge University Press, 2004.

[27] S. P. Boyd. (2008) Subgradient methods (lecture notes). [Online]. Available: http://www.stanford.edu/class/ee364b/lectures/ subgrad_method_slides.pdf

[28] 3GPP TS 36.216 V10.3.1. (2011) Universal mobile telecommunications system (UMTS);evolved universal terrestrial radio access (E-UTRA); physical layer for relaying operation. [Online]. Available: http: //www.3gpp.org/ftp/Specs/html-info/36216.htm

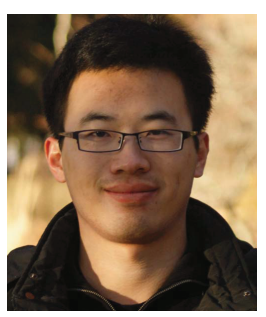

Honghao Ju is currently a Ph.D. student at Xidian University, Xi'an, China, where he received his BE degree in telecommunication engineering in 2009. During 2012-2013, he was a visiting student at the University of Toronto. His research interests are stochastic network optimization, network architecture analysis, and optimization techniques in the network design.

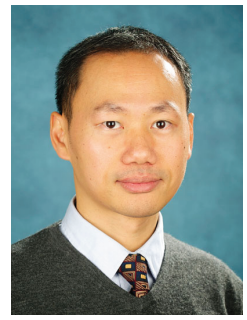

Ben Liang received honors-simultaneous B.Sc. (valedictorian) and M.Sc. degrees in Electrical Engineering from Polytechnic University in Brooklyn, New York, in 1997 and the Ph.D. degree in Electrical Engineering with Computer Science minor from Cornell University in Ithaca, New York, in 2001. In the 2001 - 2002 academic year, he was a visiting lecturer and post-doctoral research associate at Cornell University. He joined the Department of Electrical and Computer Engineering at the University of Toronto in 2002, where he is now a Professor. His research interests are in mobile communications and networked systems. He received an Intel Foundation Graduate Fellowship in 2000 and an Ontario MRI Early Researcher Award (ERA) in 2007. He was a co-author of the Best Paper Award at the IFIP Networking conference in 2005. He is an editor for the IEEE Transactions on Wireless Communications and an associate editor for the Wiley Security and Communication Networks journal, in addition to regularly serving on the organizational or technical committee of a number of conferences. He is a senior member of IEEE and a member of ACM and Tau Beta Pi. 


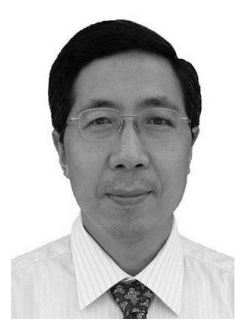

Jiandong $\mathbf{L i}$ received the BE, MS, and Ph.D. degrees from Xidian University, Xi'an, China, in 1982, 1985, and 1991, respectively, all in electrical engineering. He has been a faculty member of Telecommunications Engineering at Xidian University since 1985 , where he is currently a professor and director of State Key Laboratory of Integrated Service Networks. Prof. Li is a senior member of IEEE. He was a visiting professor to the Department of Electrical and Computer Engineering at Cornell University from 2002- 2003. He was a member of Personal Communication Networks (PCN) specialist group for China 863 Communication High Technology Program during 1993-1994 and again 19992000. He was awarded as Distinguished Young Researcher and Changjiang Scholar from Ministry of Science and Technology, China. His major research interests are wireless communication theory, cognitive radio, and signal processing.

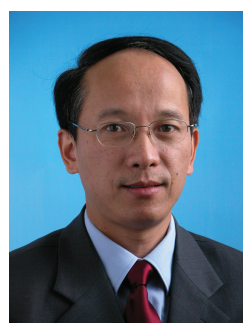

Xiaoniu Yang is a research scientist at NO.36 research institute of CETC, Jiaxing, China. He got his $\mathrm{BE}$ and MS degrees in electrical engineering from Xidian University, Xi'an, China, in 1982 and 1988, where he currently holds an adjunct professor position. His research interests are software-defined radio and signal processing. 